\title{
AQUISIÇÃO DE HABILIDADES FUNCIONAIS NA ÁREA DE MOBILIDADE EM CRIANÇAS ATENDIDAS EM UM PROGRAMA DE ESTIMULAÇÃO PRECOCE
}

\author{
ACQUISITION OF FUNCTIONAL ABILITIES IN THE MOBILITY AREA BY \\ CHILDREN ASSISTED IN AN EARLY STIMULATION PROGRAM
}

Camilla Zamfolini Hallal

Nise Ribeiro Marques*

Ligia Maria Presumido Braccialli*

Hallal CZ, Marques NR, Braccialli LMP. Aquisição de Habilidades Funcionais na Área de Mobilidade em Crianças Atendidas em um Programa de Estimulação Precoce. Rev Bras Crescimento Desenvol Hum. 2008; 18(1): 27-34.

\begin{abstract}
Resumo: A estimulação precoce visa a evitar ou minimizar os distúrbios do desenvolvimento neuropsicomotor e possibilitar à criança desenvolver-se em todo o seu potencial. O objetivo deste estudo foi analisar, segundo a percepção dos cuidadores, a aquisição de habilidades funcionais na área de mobilidade em crianças com atraso no desenvolvimento neuropsicomotor atendidas em um programa de estimulação precoce. Participaram do estudo crianças com idade de 0 e 3 anos atendidas no programa de estimulação precoce. A coleta de dados foi realizada em outubro de 2006 e maio de 2007, utilizando-se a parte I do teste Pediatric Evaluation of Disability Inventory. A análise do desempenho das crianças na área de mobilidade, de acordo com a idade cronológica, foi obtida por meio de escore padronizado normativo. Os resultados indicaram que 5 crianças apresentaram evolução, 1 criança apresentou regressão e 2 crianças mantiveram a mesma pontuação. Tal fato pode estar relacionado ao diagnóstico clínico de cada criança, os quais mantêm características específicas que influenciam de forma distinta no desenvolvimento neuropsicomotor. Conclui-se que, segundo o escore normativo, a maioria das crianças obteve evolução na pontuação. Assim, sugere-se que o programa de estimulação precoce, aliado ao desenvolvimento intrínseco da criança, contribui para a aquisição de habilidades funcionais na área de mobilidade em crianças com atraso no desenvolvimento.
\end{abstract}

Palavras-chave: Desenvolvimento motor; estimulação precoce; PEDI.

\section{INTRODUÇÃO}

O desenvolvimento motor segue uma ordem cronológica evolutiva com etapas distintas e previsíveis, caracterizadas por mudanças nas habilidades e nos padrões de movimento que ocorrem durante a vida ${ }^{1,2}$.

Estas mudanças ocorridas ao longo do desenvolvimento vêem sendo estudadas sob o enfoque de diversas teorias. Durante muitos anos, o desenvolvimento motor foi explicado pelo ponto de vista neuromaturacional, no qual as al- terações no comportamento motor se deviam exclusivamente à maturação do sistema nervoso. A teoria dos sistemas dinâmicos, mais aceita atualmente, apontou que o comportamento motor não é influenciado apenas pelo sistema nervoso, mas também por outros fatores, como os psicológicos e os ambientais ${ }^{1}$. Segundo Tecklin $(2002)^{3}$, é a interação destes fatores que promove o desenvolvimento das habilidades motoras.

O desenvolvimento neuropsicomotor da criança pode, no entanto, ser afetado negativa-

\footnotetext{
Faculdade de Filosofia e Ciências. Departamento de Educação Especial. Curso de Fisioterapia. UNESP; campus de Marília (Av. Hygino Muzzi Filho; 737; Cx Postal 181; CEP 17525-900)

Contatos com: Camilla Zamfolini Hallal: e-mail: camillazhallal@yahoo.com.br
} 
mente por diversos fatores incidentes nos períodos pré, peri e/ou pós-natais. Estes fatores aumentam a probabilidade da criança manifestar alterações na aquisição de habilidades motoras, cognitivas e psicossociais ${ }^{4,5}$. Assim, para possibilitar a intervenção precoce nos atrasos evolutivos é imprescindível a identificação de distúrbios no desenvolvimento neuropsicomotor no primeiro ano de vida ${ }^{6,7}$.

Além dos déficits neuromotores, os atrasos no desenvolvimento podem, também, resultar em limitações nas habilidades funcionais. As atividades funcionais incluem, por exemplo, atividades de auto-cuidado como alimentação e banho independentes, atividades de mobilidade como levantar da cama e ir ao banheiro com independência, além de tarefas de função social como ir à escola e interagir com outras crianças ${ }^{8}$.

A estimulação precoce visa a possibilitar ao indivíduo desenvolver-se em todo o seu potencial ${ }^{9,10}$. Quanto mais imediata for a intervenção, preferencialmente antes dos 3 anos de idade, maiores as chances de prevenir e/ou minimizar a instalação de padrões posturais e movimentos anormais ${ }^{9,11,12}$.

A intervenção precoce baseia-se em exercícios que visam ao desenvolvimento da criança de acordo com a fase em que ela se encontra ${ }^{13}$. Assim, implementa-se um conjunto de atividades destinadas a proporcionar à criança, nos primeiros anos de vida, o alcance do pleno desenvolvimento ${ }^{14}$.

As incapacidades e/ou dificuldades da criança são manifestadas durante o desempenho das atividades de vida diária. Assim, existe a necessidade de conhecer a opinião dos cuidadores sobre a aquisição das habilidades funcionais das crianças com atraso no desenvolvimento e do impacto que este gera nas rotinas diárias.

O objetivo deste estudo foi analisar a aquisição de habilidades funcionais na área de mobilidade em crianças com atraso no desenvolvimento inseridas no Programa de Estimulação Precoce do Centro de Estudos da Educação e da Saúde na Universidade Estadual Paulista, campus de Marília.

\section{MÉTODO}

\section{Participantes}

Participaram do estudo oito cuidadores de crianças de 0 a 3 anos de idade com atraso no desenvolvimento, atendidas no Programa de Estimulação Precoce do Centro de Estudos da Educação e da Saúde. Todas as crianças atendidas no Programa de Estimulação Precoce do Centro de Estudos da Educação e da Saúde em outubro de 2006 foram convidadas a participar do estudo. Foram excluídos da pesquisa os indivíduos que não se dispuseram a participar, bem como aqueles que desistiram do atendimento no período da coleta de dados, entre outubro de 2006 a maio de 2007.

\section{Considerações Éticas}

O projeto de pesquisa foi aprovado pelo comitê de ética da Faculdade de Filosofia e Ciências, da Universidade Estadual Paulista, campus de Marília (parecer n 2691/2006). Todos os participantes do estudo assinaram o termo de consentimento livre e esclarecido, de acordo com as resoluções 196/96 e 257/97.

\section{Intrumento}

Para a coleta de dados foi utilizado o teste funcional norte-americano Pediatric Evaluation of Disability Inventory (PEDI), o qual foi traduzido e adaptado às condições socioculturais brasileiras. O PEDI foi desenvolvido com o objetivo de fornecer informações detalhadas sobre o desempenho funcional da criança, predizer seu desempenho futuro e documentar mudanças no desempenho funcional.

A avaliação com o questionário PEDI é feita por meio de entrevista ao cuidador, o qual deve saber informar sobre o desempenho da criança em atividades e tarefas típicas da rotina diária. Este teste avalia os aspectos funcionais do desenvolvimento de crianças com idade entre 6 meses e 7 anos e meio, mas também pode ser utilizado na avaliação de crianças com idade superior, porém, com desempenho funcional den- 
tro da faixa etária proposta.

O teste é composto por três partes distintas. A primeira parte avalia as habilidades funcionais da criança, as quais são agrupadas em três aspectos do desenvolvimento: auto-cuidado (73 itens), mobilidade (59 itens) e função social (65 itens). Cada item desta parte é pontuado com escore 0 se a criança não é capaz de realizar a atividade funcional, ou 1 se a atividade já fizer parte do repertório de habilidades funcionais da criança. Os 59 itens avaliados na escala de mobilidade são subdivididos nas tarefas de transferências (24 itens), locomoção em ambientes internos (13 itens), locomoção em ambientes externos (12 itens), e uso de escadas (10 itens).

A segunda parte do teste PEDI avalia a quantidade de assistência fornecida pelo cuidador à criança no desempenho das atividades funcionais nas áreas de auto-cuidado, mobilidade e função social. Na terceira parte do PEDI são documentadas as modificações do ambiente usadas pela criança no desempenho das habilidades funcionais das áreas de auto-cuidado, mobilidade e função social.

\section{Procedimento para coleta de dados}

A coleta de dados foi feita por meio de entrevista direta aos cuidadores durante visitas realizadas na residência destes. As visitas foram agendadas com antecedência e os participantes informados sobre o objetivo da mesma.

As primeiras visitas ocorreram em outubro de 2006 nas quais os cuidadores responderam pela primeira vez ao teste PEDI. Seis meses depois, em maio de 2006, foram realizadas novas visitas, e os cuidadores responderam novamente ao teste PEDI. No período entre outubro de 2006 e maio de 2007 todas as crianças com atraso no desenvolvimento neuropsicomotor foram atendidas duas vezes por semana no programa de estimulação precoce do Centro de Estudos da Educação e da Saúde.

\section{Procedimentos para a análise dos resultados}

Para este estudo foram utilizados os dados obtidos na área de mobilidade da Parte I, habilidades funcionais, do teste PEDI. Um escore bruto foi obtido como resultado da somátoria de todos os itens pontuados na área de mobilidade, que informava sobre o desempenho da criança nesta área.

Pela análise estatística de Rash, a partir dos escores brutos e da idade cronológica das crianças foram obtidos os escores normativos que informaram sobre o desempenho esperado de crianças de mesma faixa etária, com desenvolvimento normal. Em cada grupo etário, o intervalo de normalidade compreende um escore normativo entre 30 e 70. Escores normativos inferiores a 30 ilustram significante atraso em relação às crianças de mesma faixa etária; escores normativos superiores a 70 ilustram um desempenho significantemente superior às crianças de mesma faixa etária; e um escore normativo de 50 corresponde ao escore médio esperado para cada grupo etário ${ }^{15}$.

Os escores normativos obtidos em outubro de 2006 e em maio de 2007 foram dispostos em tabelas para análise dos resultados e discussão.

\section{RESULTADOS E DISCUSSÃO}

Na Tabela 1 são apresentados os resultados referentes à caracterização das crianças às quais se refere o questionário PEDI, respondido pelos participantes da pesquisa.

A Tabela 2 mostra o desempenho das crianças na área de mobilidade no período compreendido entre outubro de 2006 e maio de 2007, segundo a percepção de seus cuidadores.

Observou-se que, no período da primeira coleta, o participante $\mathrm{C} 1$ obteve um escore normativo abaixo do intervalo de normalidade que compreende um escore normativo entre 30 e 70. No período da segunda coleta, maio de 2007, o escore normativo continuou com o mesmo valor, portanto ainda se encontrava abaixo do intervalo de normalidade.

A criança $\mathrm{C} 1$ foi encaminhada ao programa de estimulação precoce do CEES com o diagnóstico clínico de paralisia cerebral diparética. Segundo Mancini et al. $(2002)^{8}$, a paralisia cerebral, também denominada encefalopatia crônica não progressiva, caracteriza-se por distúr- 
Tabela 1 - Caracterização das crianças cuidadas pelos participantes.

\begin{tabular}{llll}
\hline Crianças & Idade cronológica (outubro de 2006) & Idade cronológica (maio de 2006) & Diagnóstico \\
\hline C1 & 3 anos e 11 meses & 4 anos e 5 meses & paralisia cerebral diparética \\
C2 & 3 anos e 5 meses & 3 anos e 11 meses & deficiência visual \\
C3 & 2 anos e 4 meses & 2 anos e 10 meses & seqüela de hidrocefalia \\
C4 & 2 anos e 11 meses & 3 anos e 5 meses & seqüela de hidrocefalia \\
C5 & 2 anos e 6 meses & 3 anos & síndrome de Dandy-Walker \\
C6 & 3 anos e 10 meses & 4 anos e 4 meses & síndrome de Jacobsen \\
C7 & 7 meses & 1 ano e 1 mês & atraso no desenvolvimento \\
C8 & 1 ano e 11 meses & 2 anos e 5 meses & atraso no desenvolvimento \\
C9 & 2 anos e 10 meses & 3 anos e 4 meses & lesão nervosa periférica \\
\hline
\end{tabular}

Tabela 2 - Valores dos escores normativos obtidos pelas crianças na área de mobilidade.

\begin{tabular}{lllllllll}
\hline & C 1 & C 2 & C 3 & C 4 & C 5 & C 6 & C 7 & C 8 \\
\hline Outubro de 2006 & $<10$ & $<10$ & $<10$ & $<10$ & $<10$ & $<10$ & 17,6 & 33,9 \\
Maio de 2007 & $<10$ & 14,8 & 25,1 & 36,2 & $<10$ & 27,9 & 16,5 & 46,5 \\
\hline
\end{tabular}

bio permanente do tônus, da postura e do movimento, causado por agressão encefálica ocorrida no período pré, peri ou pós-natal. O comprometimento motor da paralisia cerebral pode envolver partes distintas do corpo com seqüelas de classificação topográfica específicas, como a quadriplegia, a diplegia e a hemiplegia.

A paralisia cerebral ocorre no período em que a criança apresenta acelerado ritmo de desenvolvimento. Assim, a paralisia cerebral pode comprometer a aquisição dos marcos motores básicos como o rolar, o sentar, o engatinhar e o andar. As dificuldades encontradas no controle do movimento interferem no desempenho das habilidades funcionais. Estas dificuldades provocam limitações nas atividades de vida diária da criança e de sua família ${ }^{8,16}$.

Existe também uma relação forte e direta entre o grau de severidade da paralisia cerebral e o desempenho funcional da criança. Quanto mais grave for a paralisia cerebral, maior será o comprometimento motor e, conseqüentemente, haverá maior dificuldade da criança em desempenhar as habilidades funcionais ${ }^{16}$.

Segundo Allegretti et al. $(2004)^{17}$, as crianças com paralisia cerebral diparética apresentam desempenho consideravelmente inferior ao das crianças da mesma faixa etária na área de mobilidade. Os resultados encontrados neste estudo corroboram com os dados da literatura, uma vez que $\mathrm{C} 1$ obteve escores normativos abaixo da faixa mínima esperada para a idade (30), em ambas as coletas, na área de mobilidade. Este valor indicou que $\mathrm{C} 1$ tinha importante atraso nesta habilidade.

Quanto ao desempenho do participante C2 na área de mobilidade no período do estudo, observou-se que este paciente obteve, na época da primeira e da segunda coleta, escores normativos inferiores a 30 , portanto abaixo do intervalo de normalidade.

$\mathrm{O}$ diagnóstico clínico de $\mathrm{C} 2$ era deficiência visual. Segundo Malta et al. (2006) ${ }^{18}$, a deficiência visual impõe restrições à capacidade de exploração ambiental livre e segura, o que dificulta a construção do conhecimento e da aprendizagem plena.

Oliveira e Marque (2005) ${ }^{11}$ descreveram que a deficiência visual, ou seja, a ausência de informações visuais é responsável por alterações no desenvolvimento da criança, como por exemplo, atrasos no desenvolvimento motor e cognitivo. Muitas habilidades funcionais são aprendidas por meio da imitação e da estimulação visual, portanto, a ausência ou deficiência da visão interfere negativamente neste processo.

De acordo com Malta et al. (2006) ${ }^{18}$, as crianças com deficiência visual apresentam maior dificuldade na área de mobilidade. Estas informações coincidiram com os achados da pesqui- 
sa, uma vez que o participante C2 apresentou, apesar da evolução, escores normativos inferiores à faixa de normalidade mínima esperada para a idade (30), caracterizando importante atraso na área de mobilidade.

O participante $\mathrm{C} 3$ obteve na primeira e na segunda coleta escores normativos inferiores a 30 na área de mobilidade, enquanto o participante $\mathrm{C} 4$ obteve na primeira coleta um escore normativo inferior a 30 e na segunda coleta um escore superior a 30 .

Os participantes C3 e C4 foram encaminhados ao programa de estimulação precoce do CEES com o diagnóstico clínico de hidrocefalia. A hidrocefalia é um importante problema médico e social, caracterizada por aumento do perímetro cefálico decorrente de distúrbios da circulação do líquido cefalorraquidiano ${ }^{19,20,21}$. O quadro clínico da hidrocefalia é bastante variável, entre outros a criança pode apresentar atraso no desenvolvimento motor, retardo mental, paraplegia espástica e aumento dos reflexos tendíneos ${ }^{21,22}$.

$\mathrm{O}$ escore normativo abaixo da faixa de normalidade mínima esperada para a idade (30) na área de mobilidade do participante $\mathrm{C} 3$ pode estar relacionado ao comprometimento motor apresentado pela criança, como relataram Jucá et al. (2002) $)^{22}$. Apesar do participante C3 continuar abaixo do intervalo de normalidade, após a segunda entrevista, os valores obtidos indicam que houve uma importante evolução na área de mobilidade. Esta evolução pode estar relacionada aos constantes estímulos motores oferecidos pela fisioterapia no programa de estimulação precoce do CEES, como também, ao desenvolvimento característico da fase em que a criança se encontra.

Da mesma forma, o participante $\mathrm{C} 4$ obteve importante evolução na área de mobilidade, pois na primeira coleta foi identificado valor abaixo da faixa de normalidade mínima esperada para a idade e na segunda coleta a criança já obteve valor dentro da faixa de normalidade esperada para a idade.

Em relação ao desempenho do participante C5 na área de mobilidade no período do estudo verificaram-se na primeira e na segunda coleta escores normativos inferiores a 30 .
A criança C5 foi encaminhada ao programa de estimulação precoce do CEES com o diagnóstico clínico de Síndrome de Dandy Walker. Segundo Pereira, et al. $(2001)^{23}$, a Síndrome de Dandy Walker é caracterizada por dilatação cística do quarto ventrículo, hipoplasia do vermis cerebelar e hidrocefalia. Esta síndrome pode manifestar-se clinicamente por atraso no desenvolvimento neuropsicomotor, hipotonia, retardo mental, convulsões, entre outros ${ }^{24}$.

$\mathrm{Na}$ área de mobilidade C5 obteve pontuação inferior à faixa de normalidade mínima esperada para a idade em ambas as coletas. Estes achados indicaram que a criança tinha um importante atraso nesta área. O baixo desempenho de C5 pode estar relacionado às características específicas da Síndrome de Dandy Walker, como relatado por Ewald et al. (2006) ${ }^{24}$.

O participante $\mathrm{C} 6$ obteve na primeira e na segunda coleta escores normativos com valores inferiores a 30. Este participante tinha diagnóstico clínico de Síndrome de Jacobsen. Esta síndrome, também conhecida como monossomia do $11 q$, apresenta como manifestações típicas atraso no desenvolvimento neuropsicomotor, o dismorfismo facial, o retardo mental, entre outros ${ }^{25}$.

Estes dados indicaram, apesar da evolução, um importante atraso no desenvolvimento da criança em relação ao esperado para crianças de mesma faixa etária. Assim, apesar das intervenções realizadas na estimulação precoce com o objetivo de maximizar o desempenho da criança nas habilidades funcionais, observou-se que as limitações da criança relacionadas à síndrome consistem em importante obstáculo ao seu pleno desenvolvimento.

A análise dos dados do participante C7 indicou que ele obteve na primeira e na segunda coleta escores normativos com valores inferiores a 30. Esta criança tinha como diagnóstico clínico atraso no desenvolvimento neuropsicomotor. Em relação ao participante C7 observou-se que o valor normativo obtido no período da segunda coleta foi menor do que o encontrado na primeira coleta. Assim, apesar dos estímulos extrínsecos oferecidos à criança na Estimulação Precoce com objetivo de desenvolver as habilidades motoras, o atraso considerável da criança 
não foi suprimido.

O participante $\mathrm{C} 8$ obteve na primeira e na segunda coleta escores com valores normativos superiores a 30 . Os resultados indicaram que $\mathrm{C} 8$ tinha um atraso considerado leve, uma vez que na primeira e na segunda coleta obteve escores normativos dentro da faixa de normalidade esperada para a sua faixa etária (entre 30 e 70). Esta criança tinha diagnóstico de atraso no desenvolvimento neuropsicomotor.

Observou-se uma evolução no quadro motor de C8, uma vez que este já conseguiu desempenhar as habilidades funcionais na área de mobilidade de forma satisfatória, ou seja, compatível com o esperado para crianças da mesma faixa etária.

Destaca-se, assim, a importância da estimulação precoce, em conjunto com fatores intrínsecos, no desenvolvimento das habilidades funcionais por meio de estímulos oferecidos pelas mais diversas áreas de atendimento visando à independência nas atividades de vida diária.

Ao se analisar a aquisição de habilidades funcionais de crianças com atraso no desenvolvimento e sugerir a contribuição da intervenção em estimulação precoce nestas aquisições, devese considerar os diversos fatores que podem contribuir para interpretação fidedigna dos resultados.

As crianças com ou sem alterações no desenvolvimento, têm influências do processo neuromaturacional na aquisição de habilidades funcionais, como relatado por Mancini et al. $(2002)^{8}$. Assim, sugere-se que a estimulação precoce contribui para o desenvolvimento neuropsicomotor, embora não atue isoladamente neste processo.

De acordo com Mancini et al. $(2002)^{8}$, a expectativa dos pais e cuidadores interfere diretamente na percepção destes em relação ao desempenho da criança. Deste modo, alguns comportamentos podem ser omitidos ou valorizados durante a avaliação na tentativa de transparecer o desempenho almejado.

Os fatores culturais também exercem influência no desenvolvimento infantil. Mancini et al. $(2002)^{8}$ e Mancini et al. $(2004)^{16}$ relatam o perfil protecionista da cultura brasileira em rela- ção à realização das tarefas de rotina diária pelas crianças. Deste modo, mesmo que a criança apresente capacidade de realizar determinadas tarefas, muitas vezes o cuidador as faz. Isto parece acontecer especialmente nas famílias das crianças com necessidades especiais, em que os cuidadores procuram realizar o maior número de tarefas para a criança com o intuito de poupálas de esforços que consideram desnecessários. Pensando nisto, os pais e cuidadores são incentivados pelos profissionais que atuam na estimulação precoce a estimular a independência funcional da criança nas atividades de vida diárias.

Ressalta-se ainda que o desempenho das habilidades funcionais pode ser prejudicado também em situações em que a criança e o adulto estão submetidos à pressão de resultados ${ }^{26}$. Deste modo, a limitação de tempo e espaço para a realização de determinadas atividades como o banho e a refeição, por exemplo, acabam por interferir diretamente na independência funcional da criança.

Desta maneira, observou-se por meio dos escores normativos que na área de mobilidade a maioria das crianças apresentou evolução, porém algumas crianças mantiveram a mesma pontuação ou regrediram nestas áreas. Deve-se considerar que o desempenho satisfatório na área de mobilidade requer o uso de habilidades motoras específicas. Deste modo, observou-se que as crianças que não apresentaram evolução nos escores normativos nestas áreas, foram as que possuíam um comprometimento motor mais grave de acordo com o quadro clínico.

O questionário PEDI mostrou-se eficaz para os objetivos propostos neste estudo pois caracterizou de forma satisfatória o perfil funcional na área de mobilidade das crianças com atraso no desenvolvimento inseridas no programa de estimulação precoce do CEES. Assim foi considerado um instrumento bastante útil na avaliação e na intervenção dos profissionais, além de possibilitar uma análise crítica do trabalho desenvolvido. Os resultados apontados no presente estudo fornecem subsídios para afirmar que a intervenção precoce pode ser eficaz na potencialização do desenvolvimento de crianças com atraso no desenvolvimento. 


\begin{abstract}
Early stimulation aims to avoid or reduce neuropsychomotor developmental disorders and to enable children to develop all their potential. The purpose of this study was to analyse, according to caregivers' perception, the acquisition of functional abilities in the mobility area by children with neuropsychomotor development delay assisted by an early stimulation program. Children aged 0 to 3 years participated in this study. The data collection was performed in October 2006 and May 2007, and part I of the Pediatric Evaluation of Disability Inventory was used. The analysis of children's performance in the mobility area, according to the chronological age, was obtained by the normative standardized score. The results showed that five children developed, one child regressed and two children maintained the same score. This fact can be related to the clinical diagnosis of each child, which maintains specific characteristics that influence neuropsychomotor development in distinct ways. We concluded that, according to the normative score, the majority of children developed. Thus, we suggest that early stimulation, together with the child's intrinsic development, contributes to the acquisition of functional abilities in the mobility area in children with delay in development.
\end{abstract}

Keywords: Motor development; early stimulation; PEDI.

\section{REFERÊNCIAS}

1. Camargos ACR, Lacerda TTB. ODesenvolvimento motor na perspectiva dos sistemas dinâmicos. Temas Sobre Desenvolvimento. 2005; 14(82):23-29.

2. Ramos C R, Lucas S, Pedromônico M R M. O desenvolvimento infantil no segundo ano de vida: existem diferenças em relação ao sexo? Temas sobre desenvolvimento. 2000; 9(53):38-43.

3. Tecklin J S. Fisioterapia Pediátrica. $3^{\mathrm{a}}$ edição. São Paulo: Artmed. 2002.

4. Caram E HA, Funayama CA R, Spina C I, Giuliani L R, Neto J M P. Investigação das causas de atraso no neurodesenvolvimento. Recursos e desafios. Arquivos de Neuropsiquiatria. 2006; 64(2B):466-472.

5. Neto F R, Caon G, Bissani C, Silva C A, Souza M, Silva L. Características neuropsicomotoras de crianças de alto risco atendidas em um programa de follow-up. Pediatria moderna. 2006; 42(2):7985 .

6. Campos D, Santos D C C, Gonçalves V M G, Goto M M F, Arias A V, Brianezi A C G S. Agreement between scales for screening and diagnosis of motor development at 6 months. Jornal de Pediatria. 2006; 82(6):470-474.

7. Bretas J R S, Pereira S R, Cintra C C, Amirati K M. Avaliação de funções psicomotoras de crianças entre 6 e 10 anos de idade. Acta Paulista de Enfermagem. 2005; 18(4):403-412.

8. Mancini M C, Fiúza P M, Rebelo J M, Magalhães L C, Coelho Z A C, Paixão M L. Comparação do desempenho de atividades funcionais em crianças com desenvolvimento normal e crianças com paralisia cerebral. Arquivos de Neuropsiquiatria.
2002; 60(2).

9. Tudella E, Formiga C K M R, Serra E L, Oish J. Comparação da eficácia da intervenção fisioterapêutica essencial e tardia em lactentes com paralisia cerebral. Fisioterapia em Movimento. 2004; 17(3):45-52.

10. Robles H S M, Williams L C A, Aiello A L R. Intervenção breve no ambiente natural de uma criança especial com família de baixo poder aquisitivo. Temas sobre Desenvolvimento. 2002; 11(63):52-57.

11. Oliveira J P, Marque S L. Análise da comunicação verbal e não verbal de crianças com deficiência visual durante a interação com a mãe. Revista Brasileira de Educação Especial. 2005; 11(3).

12. Formiga C K M R, Pedrazzani E S, Tudella E. Desenvolvimento motor de lactentes pré-termo participantes de um programa de intervenção fisioterapêutica precoce. Revista Brasileira de Fisioterapia. 2004; 8(3):239-245.

13. Sarro K J, Salina M E. Estudo de alguns fatores que influenciam no desenvolvimento das aquisições motoras de crianças portadoras de síndrome de Down em tratamento fisioterápico. Fisioterapia em Movimento. 1999; 13(1):93-103.

14. Mec. Diretrizes Educacionais Sobre Estimulação Precoce. Brasília, 1995.

15. Mancini, M. C. Inventário de avaliação pediátrica de incapacidade (PEDI) - manual da versão brasileira adaptada. Belo Horizonte: UFMG. 2005.

16. Mancini M C, Alves A C M, Schaper C, Figueiredo E M, Sampaio R F, Coelho Z A. Gravidade da paralisia cerebral e desempenho funcional. Revista Brasileira de Fisioterapia. 2004; 8(3):253260. 
17. Allegretti A LC, Mancini M C, Schwartzman J S. Estudo do desempenho functional de crianças com paralisia cerebral diparética espástica utilizando o Pediatric Evaluation of Disability Inventory (PEDI). Arquivos Brasileiros de Paralisia Cerebral. 2004; 1(1):35-40.

18. Malta J. Endriss D, Rached S, Moura T, Ventura L. Desempenho funcional de crianças com deficiência visual, atendidas no departamento de estimulação visual da Fundação Altino Ventura. Arquivos Brasileiros de Oftalmologia. 2006; 69(4).

19. Cavalcanti D P, Salomão M A. Incidência da hidrocefalia congênita e o papel do diagnóstico pré-natal. Jornal de Pediatria. 2003; 72(2).

20. Kliemann S E, Rosemberg S. Hidrocefalia derivada na infância: um estudo epidemiológico de 243 observações consecutivas. Arquivos de Neuropsiquiatria. 2005; 63(2).

21. Vieira M W, Cavalcanti D P, Lopes V L G S. Importância da avaliação genético-clínica na hidrocefalia. Arquivos de Neuropsiquiatria. 2004; 62(2).
22. Jucá C E B, Neto, AL, Oliveira R S, Machado H R. Tratamento da hidrocefalia com derivação ventrículo-peritoneal: análise de 150 casos consecutivos do Hospital das Clínicas de Ribeirão Preto. Acta Cirúrgica Brasileira. 2002; 17(3).

23. Pereira C U, Machado S C. Síndrome de DandyWalker. Revista Brasileira de Medicina. 2001; 58(5):348-349.

24. Ewald O, Scremin F Busch, F Hertwig R V Alterações oculares em paciente pediátrico portador de malformações de Dandy-Walker: relato de caso. Arquivos Brasileiros do Oftalmolgia 2006; 69(1).

25. Santos D V V. Frasson, M, Caldas E D;Leao, L; AguiaR, M. J. B. Monossomia do 11q com coloboma ocular: relato de um caso. Revista Brasileira de Oftalmologia; 2002; 61(9):682-685.

26. Oliveira M C, Cordani L K. Correlação entre habilidades funcionais referidas pelo cuidador $\mathrm{e}$ o nível de assistência fornecida a crianças com paralisia cerebral. Arquivos Brasileiros de Paralisia Cerebral, 2004; 1(1):24-29.

Recebido em: 16/07/2007

Modificado em: 22/02/2008

Aprovado em: 29/03/2008 